\title{
Peripheral blood monocytes predict clinical prognosis and support tumor invasiveness through NF-кB-dependent upregulation of Snail in pancreatic cancer
}

\author{
Shaojie Chen ${ }^{1,2 \#}$, Feifei Huang ${ }^{3 \#}$, Chong $\mathrm{He}^{1,2 \#}$, Jiajia $\mathrm{Li}^{4}$, Shangxiang Chen ${ }^{5}$, Yaqing $\mathrm{Li}^{1,2}$, Yinting Chen ${ }^{1,2}$, \\ Guoda Lian $^{1,2}$, Kaihong Huang ${ }^{1,2} \wedge$ \\ ${ }^{1}$ Guangdong Provincial Key Laboratory of Malignant Tumor Epigenetics and Gene Regulation, Sun Yat-sen Memorial Hospital, Sun Yat-sen \\ University, Guangzhou, China; ${ }^{2}$ Department of Gastroenterology, Sun Yat-sen Memorial Hospital, Sun Yat-sen University, Guangzhou, China; \\ ${ }^{3}$ Department of Cardiology, Sun Yat-sen Memorial Hospital, Sun Yat-sen University, Guangzhou, China; ${ }^{4}$ Department of Nephrology, Sun Yat-sen \\ Memorial Hospital, Sun Yat-sen University, Guangzhou, China; ${ }^{5}$ Department of Gastrointestinal Surgery, Sun Yat-sen Memorial Hospital, Sun Yat- \\ sen University, Guangzhou, China \\ Contributions: (I) Conception and design: G Lian, K Huang; (II) Administrative support: Y Chen, K Huang; (III) Provision of study materials or \\ patients: S Chen, F Huang, C He; (IV) Collection and assembly of data: S Chen, F Huang, J Li, S Chen; (V) Data analysis and interpretation: S \\ Chen, C He, Y Li, G Lian; (VI) Manuscript writing: All authors; (VII) Final approval of manuscript: All authors. \\ \#These authors contributed equally to this work. \\ Correspondence to: Guoda Lian; Kaihong Huang. Department of Gastroenterology, Sun Yat-sen Memorial Hospital, Sun Yat-sen University, No. 107 \\ Yanjiang West Road, Guangzhou 510120, China. Email: liangd28@mail.sysu.edu.cn; huangkh@mail.sysu.edu.cn.
}

Background: The tumor inflammatory microenvironment plays a vital role in the initiation and progression of pancreatic cancer (PC). Both the lymphocyte-to-monocyte ratio (LMR) and preoperative peripheral blood monocytes are related to the prognosis of PC patients. However, the direct effect of monocytes on PC cells is not fully understood. The current study aimed to assess the effect of monocytes on PC and explore its potential mechanism.

Methods: The cutoff value of peripheral blood monocytes was evaluated by the receiver operating characteristic (ROC) curve. Transwell migration and invasion assays were used to detect the mobility of PC cells. The cytokines derived from monocytes were measured by quantitative real-time polymerase chain reaction (qRT-PCR). Western blotting was utilized to assess the expression of epithelial-mesenchymal transition (EMT) related markers. The expression level of Snail in PC tissue was determined by immunohistochemical (IHC) staining.

Results: A high monocyte count was inversely correlated with lymph node status and 5-year overall survival in PC. The PC cells underwent a cellular morphology change and increased cell motility after coculture with THP-1 monocytes. The THP-1 monocytes secreted various proinflammatory cytokines, including tumor necrosis factor- $\alpha(\mathrm{TNF}-\alpha)$ and interleukin- $1 \alpha(\mathrm{IL}-1 \alpha)$, which activated the nuclear factor- $\kappa \mathrm{B}(\mathrm{NF}-\kappa \mathrm{B})$ signaling pathway leading to the upregulation of Snail and thereby promoting the EMT of PC cells. The expression level of Snail correlated significantly with the density of peripheral blood monocytes, and their level status was significantly associated with 5-year overall survival.

Conclusions: These findings indicated that elevated monocytes counts were a poor prognostic marker in PC, and monocytes could directly induce the EMT process of PC cells by upregulating Snail expression through the NF- $\mathrm{KB}$ signaling pathway.

Keywords: Pancreatic cancer (PC); peripheral blood monocytes; tumor necrosis factor- $\alpha$ (TNF- $\alpha$ ); Snail; epithelial-mesenchymal transition (EMT)

$\wedge$ ORCID: 0000-0002-1164-8115. 
Submitted Jun 08, 2021. Accepted for publication Sep 09, 2021.

doi: $10.21037 /$ tcr-21-980

View this article at: https://dx.doi.org/10.21037/tcr-21-980

\section{Introduction}

Pancreatic ductal adenocarcinoma (PDAC), often called pancreatic cancer (PC), is the most lethal solid tumor with a 5 -year survival rate of $10 \%$ and a median survival time of only 6 months in the United States (1). PC has been ranked the 7th and 11th most common cancer in China for males and females, respectively, and the 6th leading cause of cancerrelated deaths (2). Of great concern is that the incidence and mortality of PC are continuing to increase due to changes in dietary composition and habits, with a diet high in fat and meat and low in vegetables and folate (3). Therefore, there are unquestionably urgent, and unmet medical needs to further improve our understanding of tumor biology, identify new biomarkers, and develop novel therapeutic targets for improving the treatment of PC.

The tissue of PC is characterized by a uniquely dense stroma that contributes to the tumor development and chemoresistance (4), and within the tumor stroma, abundant immune cells such as monocytes/macrophages, lymphocytes and fibroblasts are present (5). Recently, increasing evidence has demonstrated that a low lymphocyte-to-monocyte ratio (LMR) or high level of circulating monocytes exert an adverse prognostic and therapeutic effect on PC $(6,7)$. Monocytes are produced and stored in the bone marrow and are recruited to the tumor site by different chemokines produced by cancer or stromal cells (8). Various studies have shown that monocytes infiltrated in tumor niches could be polarized as a new immunosuppressive subpopulation of cells termed monocytic-myeloid derived suppressor or tumor-associated macrophages (TAMs), both of which have been shown to play a pivotal role in the progression of PC $(9,10)$. However, the direct implication of monocytes on PC cells is not clearly understood.

This study found that high monocyte count was closely associated with lymph node metastasis and the short survival of patients with PC. To determine how monocytes promote PC progression, we cocultured monocytic cell line THP-1 with PC cells. The results showed that THP-1 monocytes could promote the migration and invasion of PC cells, the mechanism of which was THP-1 monocytes mediated epithelial-mesenchymal transition (EMT) in cancer cells via upregulating Snail expression. In summary, we may provide a new possibility for the link between monocyte and PC biology. We present the following article in accordance with the MDAR reporting checklist (available at https://dx.doi. org/10.21037/tcr-21-980).

\section{Methods}

\section{Cell cultures}

Human PC cells lines PANC-1 and BxPC-3 were purchased from the American Type Culture Collection (ATCC, Manassas, VA, USA), while monocytoid THP-1 was obtained from the Cell Bank at the China Academy of Science (Shanghai, China). All cells were maintained in a humidified atmosphere of $5 \% \mathrm{CO}_{2}$ at $37{ }^{\circ} \mathrm{C}$ in Roswell Park Memorial Institute (RPMI, Buffalo, NY, USA) 1,640 media supplemented with $10 \%$ fetal bovine serum (FBS; Biological Industries, Beit Haemek, Israel). For coculture experiment, $1 \times 10^{5}$ PC cells (PANC- 1 and BxPC- 3 ) in $2 \mathrm{~mL}$ medium were seeded into a 6 -plate well, and $2 \times 10^{5}$ THP- 1 monocytes in $1.5 \mathrm{~mL}$ medium were seeded into a Transwell insert $(0.4 \mu \mathrm{m}$, Corning Costar, Lowell, MA, USA), which was placed into the well with PC cells. After cocultured for $72 \mathrm{~h}, \mathrm{PC}$ cells were trypsinized with trypsin-EDTA, washed and resuspended in complete culture medium for further experiments. The nuclear factor- $\kappa \mathrm{B}(\mathrm{NF}-\kappa \mathrm{B})$ inhibitor, BAY11-7082, was purchased from the Beyotime Institute of Biotechnology (Shanghai, China), and PC cells were pretreated for $2 \mathrm{~h}$ with the concentration of $10 \mu \mathrm{mol} / \mathrm{L}$ before proceeding to the next steps.

\section{Patients and participants}

This retrospectives study was approved by the Ethics Committee of Sun Yat-sen Memorial Hospital, Sun Yatsen University (Guangzhou, China) and individual consent for this retrospective analysis was waived. From September 2004 to December 2011, the data of 70 PC patients who underwent pancreatic resections were retrieved, and their demographic data, serum tumor marker, pathologic features of the tumor, monocyte count, and survival time were recorded for statistical analysis. All blood tests were performed preoperatively. 


\section{Migration and invasion assays}

Migration and invasion assays were performed using the PANC-1 and BxPC-3 cells in 24-well plates using polycarbonate membrane Transwell inserts $(8 \mu \mathrm{m}$, Corning Costar, Lowell, MA, USA) with and without Matrigel (Becton, Dickinson, and Co. Biosciences, Franklin Lakes, NJ, USA), respectively. Also, $2 \times 10^{4}$ cells/well of each cell line were resuspended in $150 \mu \mathrm{L}$ of serum-free culture medium and placed in the upper chamber of the Transwell insert. Then, the inserts were placed in a 24 -well culture dish filled with $500 \mu \mathrm{L}$ of the standard medium of $5 \%$ FBS, which was used as a chemoattractant. Cells were allowed to progress at $37{ }^{\circ} \mathrm{C}$ in a humidified $5 \% \mathrm{CO}_{2}$ environment for 15 and $36 \mathrm{~h}$ for migration and invasion assays. The non-migrated cells were carefully removed from the upper surface of the Transwell membrane using a cotton swab; then the penetrating cells were fixed with $4 \%$ paraformaldehyde and stained with $0.05 \%$ crystal violet. The stained cells were quantified in 5 random fields under a microscope (Nikon, Tokyo, Japan) (100× magnification).

\section{Western blotting}

Total cell lysates were harvested with protease inhibitors in radioimmunoprecipitation assay (RIPA) buffer (Beyotime, Shanghai, China). Equal amounts of protein lysates were separated in $10 \%$ sodium dodecyl sulfate-polyacrylamide gel electrophoresis (SDS-PAGE) by electrophoresis and transferred to polyvinylidene fluoride (PVDF) membranes by electroblotting. Sequentially, the membranes were incubated with different primary antibodies and horseradish peroxidase (HRP)-conjugated secondary antibody. The protein bands were detected by enhanced chemiluminescence (ECL). Glyceraldehyde 3-phosphate dehydrogenase (GAPDH) was used as the loading control. The primary antibodies were used as follows: anti-E-cadherin (1:1,000, Cell Signaling Technology, Danvers, MA, USA); anti-vimentin (1:1,000, Cell Signaling Technology, Danvers, MA, USA); anti-Snail (1:1,000, Santa Cruz Biotechnology, Santa Cruz, CA, USA); anti-NF-кB (p65) (1:1,000, Cell Signaling Technology, Danvers, MA, USA), and anti-GAPDH (1:1,000, Cell Signaling Technology, Danvers, MA, USA).

\section{Quantitative real-time polymerase chain reaction (qRT-PCR)}

Total RNA was extracted from THP-1 monocytes by
Trizol reagent (Invitrogen, Carlsbad, CA, USA), and complementary DNA (cDNA) was synthesized with a reverse transcription system kit (Takara, Dalian, China). qRT-PCR was performed on LightCycler480 Realtime PCR System (Roche, Basel, Switzerland). All primers used for qRT-PCR are listed in Table S1.

\section{Immunobistochemistry and evaluation}

As previously described, formalin-fixed, paraffin-embedded PC tissues were cut into $3 \mu \mathrm{m}$ sections for histological analysis (11). Briefly, the sections were deparaffinized in xylene, rehydrated with graded ethanol, and blocked endogenous peroxidase activity by incubating the sections in $0.3 \% \mathrm{H}_{2} \mathrm{O}_{2}$. After blocking nonspecific binding, the sections were incubated with a primary antibody directed against Snail (1:200, Santa Cruz Biotechnology, Dallas, TX, USA) overnight at $4{ }^{\circ} \mathrm{C}$. Then, the sections were incubated with secondary antibody conjugated with HRP for $1 \mathrm{~h}$ at room temperature. After washing with Tris-buffered saline solution, the sections were incubated in diaminobenzidine solution for $5 \mathrm{~min}$ and counterstained with hematoxylin for $1 \mathrm{~min}$. The intensity of immunostaining was evaluated and scored as described previously (12) by 2 independent observers who were blinded to the participants' clinical features. The scoring standard was as follows: 0 , no positive cells detectable; 1 , very few positive cells $(<5 \%)$ showing weak light-brown staining; 2 , a few positive cells $(<10 \%)$ showing weak to medium brown staining; 3 , a few positive cells $(<10 \%)$ showing medium to dark brown staining; 4 , a large number of positive cells $(>10 \%)$ showing medium to dark brown staining; 5 , a large number of positive cells (>20\%) exhibiting medium to dark brown staining. The expression level of Snail was defined as low with a score of $0-2$ and $3-5$ as high, respectively.

\section{Statistical analysis}

Participant characteristics were presented as median (range), and the participant number of each pathological factor was listed. The optimal cutoff value of peripheral blood monocyte count was determined by performing a receiver operating characteristic (ROC) curve analysis. The participants were classified into a high and low monocyte group based on the obtained optimal threshold value. The chi-square test was used to assess the difference between the peripheral blood monocyte count and clinicopathological characteristics. The Kaplan-Meier method was used to 
evaluate the event-time distribution of 5 -year overall survival, and the log-rank test was used to estimate significance. The experimental data are presented as the mean $\pm \mathrm{SD}$. The student's $t$-test was used to analyze the statistical significance between 2 groups, and a one-way analysis of variance (ANOVA) test was used for multiple comparisons. The correlation of peripheral blood monocyte count with Snail expression level [immunohistochemical (IHC) staining] was analyzed using the Spearman rank correlation coefficient. All the statistical analyses were 2 -sided, and a $\mathrm{P}$ value less than 0.05 was considered statistically significant. All statistical analyses were performed with the software SPSS 19.0 (IBM Corp., Armonk, NY, USA).

The study was conducted in accordance with the Declaration of Helsinki (as revised in 2013).

\section{Results}

\section{Participant characteristics}

Of the 70 enrolled PC patients who underwent pancreatectomy, there were 26 males and 44 females, at an average age of $59.06 \pm 11.34$ years. The median follow-up duration for the entire cohort was 11 months (range, 1 to 60 months). The mean and median preoperative serum CA199 levels were $1,394.74$ and 317.55 (range, 0.50 to $16,800.00$ ), respectively, and those of carcinoembryonic antigen (CEA) levels were 10.62 and 3.20 (range, 0.40 to 167.30), respectively. Increased CA199 (>34.00 U/mL) and CEA level $(>5.00 \mathrm{ng} / \mathrm{mL})$ were found in $49(70.00 \%)$ and $23(32.86 \%)$ cases, respectively. The majority of participants were diagnosed with tumor size no less than $3 \mathrm{~cm}$ (48/70, 68.57\%) and had positive lymph node metastases (41/70, 58.57\%), henceforth based on the tumor, node, metastasis (TNM) staging methods according to the current American Joint Committee on Cancer (AJCC) classification criteria (13), a large proportion of stage III and IV cases (57/70, 81.43\%) were observed (Table 1). However, we found that only $12(12 / 70,17.14 \%)$ participants suffered from distant metastases, the reason for which might be that most of the advanced PC patients had progressed past the surgical window at their time of diagnosis.

\section{Correlation of clinicopathological features and absolute monocyte count}

The optimal cutoff value for contrasting between the low and high monocyte groups of the peripheral blood monocyte was set to $590 / \mu \mathrm{L}$; obtained from the ROC curve analysis, which demonstrated a maximum Youden index ( $\mathrm{YI}=0.410$ ), with an area under the curve (AUC) of 0.748 [95\% confidence interval $(\mathrm{CI}): 0.634-0.861 ; \mathrm{P}<0.001$ ] (Figure 1). Therefore, the participants were classified into 2 groups, namely the low $(<590 / \mu \mathrm{L} ; \mathrm{n}=40)$ and high $(\geq 590 / \mu \mathrm{L} ; \mathrm{n}=30)$ monocyte groups, respectively. Our results demonstrated that a high monocyte count was significantly associated with lymph node metastasis $(\mathrm{P}=0.008)$ and gender $(\mathrm{P}=0.01)$ but had no association with CA199 or CEA level, tumor size and differentiation, depth of tumor invasion, and distant metastasis (Table 1). Further, the participants with high monocyte counts had a median 5-year overall survival of 7 months (95\% CI: 4.85-9.15), while those with low monocytes counts were 11 months (95\% CI: 12.23-15.77; $\mathrm{P}=0.008$ ) (Figure 2), signifying that a high absolute monocyte count was an adverse prognostic factor in PC.

\section{PC cells underwent EMT switch when cocultured with THP-1 monocytes}

Obtaining a sufficient number of human monocytes was onerous as they could not proliferate with impunity and therefore restricted their application in in vitro functional studies. Instead of human monocytes, we used immortalized proliferative cell lines (THP-1) in most of the in vitro experiments. The THP-1 monocytes are suitable alternatives to human monocytes as they retain numerous properties of the native monocyte and have rapid growth and no donorrelated variability (14). We found that the high peripheral blood monocyte count was associated with lymphatic metastasis and poor outcome. To investigate the effect of monocytes on PC, both PANC-1 and BxPC-3 cells were monoculture or indirectly cocultured with THP-1 monocyte cells using Transwell inserts, and the morphological alterations were examined under the microscope. As indicated in Figure 3A, the human PC cells (PANC-1 and BxPC-3) grew in monolayer when monoculture, similar to epithelial clusters. However, when cocultured with human monocyte THP-1 cells, they displayed spindle fibroblastic morphology, which were characterized by morphological changes from a pebble-like phenotype to an elongated phenotype. We next examined the effect of THP-1 monocytes on the migration and invasion of PC cells by Transwell-chamber assays after $72 \mathrm{~h}$ of monoculture or coculture. The 2-chamber migration and Matrigel invasion assays demonstrated that THP1 monocytes significantly promoted greater migration of 
Table 1 Correlation between the patients' clinicopathological factors and level of peripheral blood monocyte count

\begin{tabular}{|c|c|c|c|c|}
\hline Variable & $\mathrm{N}$ & \multicolumn{2}{|c|}{ Peripheral blood monocytes $(/ \mu \mathrm{L})$} & $P$ value \\
\hline Gender & & & & 0.010 \\
\hline Male & 26 & $20(50.0 \%)$ & $6(20.0 \%)$ & \\
\hline Female & 44 & $20(50.0 \%)$ & $24(80.0 \%)$ & \\
\hline$<60$ & 36 & $21(52.5 \%)$ & $15(50.0 \%)$ & \\
\hline$\geq 60$ & 34 & $19(47.5 \%)$ & $15(50.0 \%)$ & \\
\hline CA199 & & & & 0.598 \\
\hline Normal & 21 & $11(27.5 \%)$ & $10(33.3 \%)$ & \\
\hline Normal & 47 & $26(65.0 \%)$ & $21(70.0 \%)$ & \\
\hline Elevated & 23 & $14(35.0 \%)$ & $9(30.0 \%)$ & \\
\hline Grade & & & & 0.382 \\
\hline 1 & 23 & $15(37.5 \%)$ & $8(26.7 \%)$ & \\
\hline 2 & 25 & $15(37.5 \%)$ & $10(33.3 \%)$ & \\
\hline 3 & 22 & $10(25.0 \%)$ & $12(40.0 \%)$ & \\
\hline T stage & & & & 0.131 \\
\hline $\mathrm{T} 1 / 2$ & 13 & $5(12.5 \%)$ & $8(26.7 \%)$ & \\
\hline Negative & 29 & $22(55.0 \%)$ & 7 (23.3\%) & \\
\hline Positive & 41 & $18(45.0 \%)$ & $23(76.7 \%)$ & \\
\hline Distant metastasis & & & & 0.583 \\
\hline Negative & 58 & $34(85.0 \%)$ & $24(80.0 \%)$ & \\
\hline Positive & 12 & $6(15.0 \%)$ & $6(20.0 \%)$ & \\
\hline
\end{tabular}

CEA, carcinoembryonic antigen.

the PANC-1 cells to the flow chamber [migration from upper to lower chamber: $77.60 \pm 4.33$ vs. $41.00 \pm 3.79$ per high power field (HPF), $\mathrm{P}<0.001$; and invasion from upper to lower chamber: $52.66 \pm 3.71$ vs. $25.67 \pm 2.96 \mathrm{HPF}, \mathrm{P}<0.001]$ (Figure 3B,3C). Similar results for BxPC-3 cells were observed (migration: $83.00 \pm 7.23$ vs. $35.33 \pm 2.91 \mathrm{HPF}$,
$\mathrm{P}<0.001$; invasion: $22.33 \pm 1.45$ vs. $11.00 \pm 2.08 \mathrm{HPF}, \mathrm{P}<0.001$ ) (Figure 3B,3C). Simultaneously, PC cells were attracted to migrate polycarbonate membrane by conditioned medium produced from THP-1 monocytes (Figure S1). As mentioned above, we had observed that PC cells exhibited spindle-shaped or fibroblast-like morphology with less 


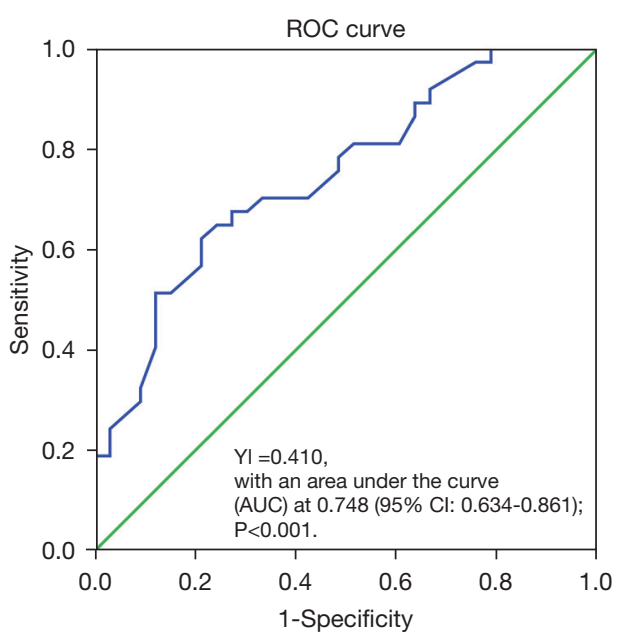

Figure 1 ROC analysis. The optimal cut-off value of monocyte was $590 / \mu \mathrm{L}$ with an AUC of 0.748 (95\% CI: 0.634-0.861; $\mathrm{P}<0.001)$. ROC, receiver operating characteristic; YI, Youden index; AUC, area under the curve; CI, confidence interval.

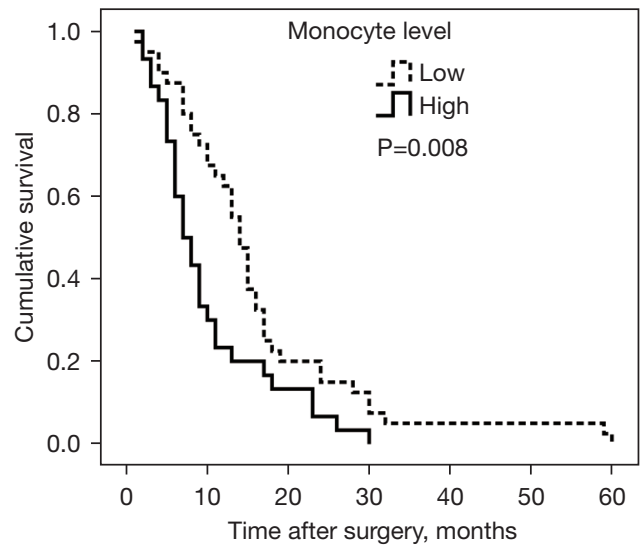

Figure 2 Survival curve according to preoperative peripheral blood monocyte count. Patients with high monocyte count $(\geq 590 / \mu \mathrm{L})$ had inferior survival rate to those with low monocyte count $(<590 / \mu \mathrm{L})$.

intense cell-to-cell contact and increased cell motility when cocultured with THP-1 monocytes. To clarify whether EMT had taken place, we tested the levels of typical molecular markers, namely E-cadherin and vimentin. As illustrated in Figure 3D, the epithelial marker E-cadherin was distinctly downregulated, and the mesenchymal marker vimentin was distinctly upregulated in PC cells. Collectively, these results suggested that THP-1 monocytes promoted the invasiveness and EMT switch of PC cells.

\section{THP-1 monocytes facilitated Snail-mediated EMT in PC cells by activating the $N F-\kappa B$ signaling patbway}

Because we chose a non-contact coculture system in our study, all kinds of cytokines were the most important inflammatory mediators between PC cells and THP-1 monocytes. We then performed a qRT-PCR assay to screen the relative expression level of a total of 12 cytokine genes, and the result indicated that the relative messenger RNA (mRNA) expression ratio of tumor necrosis factor- $\alpha$ (TNF- $\alpha$ ) to GAPDH was 79.70 , which suggested that TNF- $\alpha$ was the most abundantly expressed gene, followed with interleukin$1 \alpha$ (IL-1 $\alpha$; the relative ratio was 11.37) (Figure 4A). It is well known that both TNF- $\alpha$ and IL- $1 \alpha$ are agonists of the NF$\kappa \mathrm{B}$ signaling pathway and that the EMT regulatory factor Snail is a downstream target gene of this pathway. Western blot analysis demonstrated that the protein expression level of NF-кB (p65) and Snail was significantly increased in PC cells when treated with conditioned medium produced by THP-1 monocytes. By using the NF-кB inhibitor BAY117082, the THP-1 monocyte-induced upregulation of Snail was observed to be inhibited as well as the reversal of the EMT switch (Figure 4B). At the same time, the ability of PC cells to invade Transwell inserts decreased in the presence of BAY11-7082 (Figure 4C,4D). These results revealed that THP-1 monocytes secreted the cytokines (most likely TNF- $\alpha$ and IL- $1 \alpha$ ) to activate the NF- $\kappa B$ signaling pathway, which in turn upregulated Snail expression and induced the EMT process in PC cells.

\section{Higher expression of Snail correlated with higher absolute monocyte count and poor prognosis in $P C$}

To test whether the expression level of Snail in PC tissue and the absolute monocyte count in peripheral blood was clinically relevant, IHC staining was performed on the 70-participant PC tissue cohort. The result indicated that positive Snail staining was located primarily in the nucleus and cytoplasm of PC cells (Figure 5A). More than half of the participants demonstrated (39/70, 55.71\%) high expression (score $\geq 3$ ) of Snail, and 31 (44.29\%) participants demonstrated a low expression of Snail (score $\leq 2)$. Moreover, a significant positive correlation was displayed between the expression level of Snail and the absolute monocyte count (Spearman rank correlation coefficients, $\mathrm{r}=0.316, \mathrm{P}=0.008$, Figure 5B). Simultaneously, we used the online database Gene Expression Profiling Interactive Analysis (GEPIA; http://gepia.cancerpku.cn/index.html) (15) to perform 
A

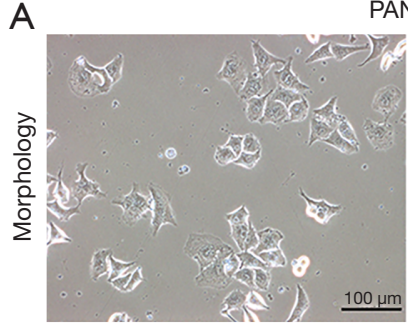

Monoculture
PANC-1

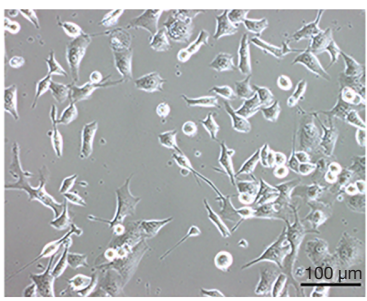

Coculture
PANC-1

B
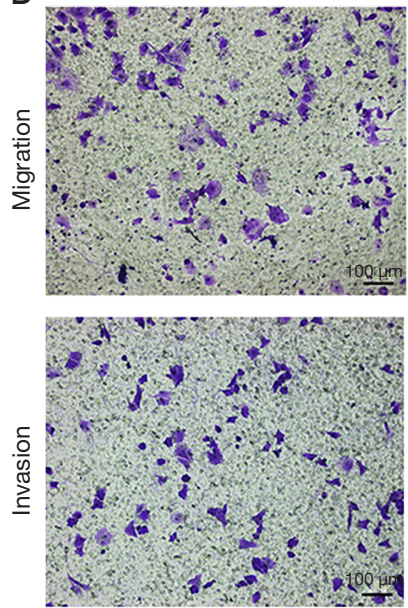

Monoculture

C

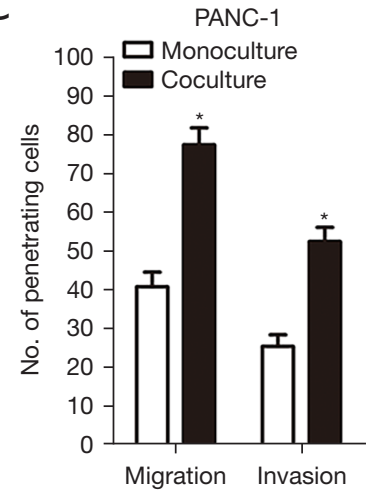

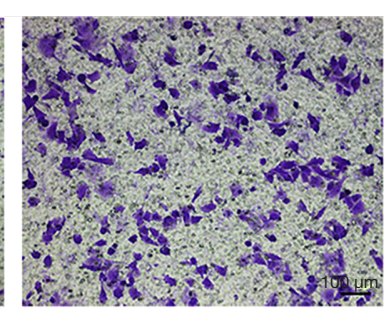

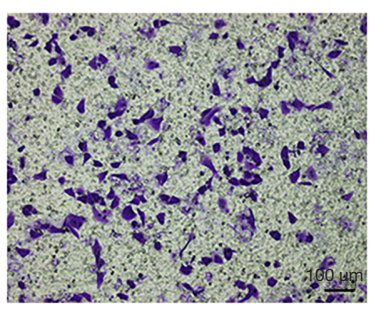

Coculture

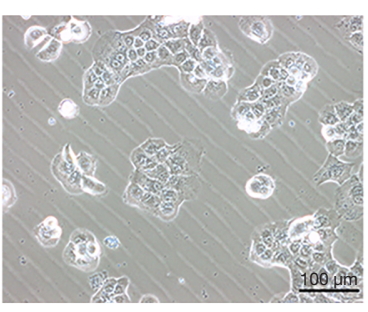

Monoculture
$\mathrm{BxPc}-3$

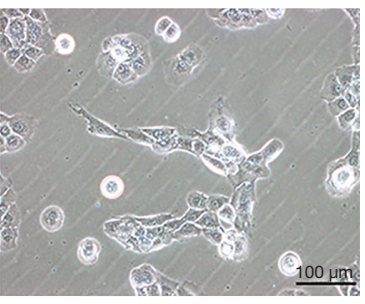

Coculture

$\mathrm{BxPc}-3$
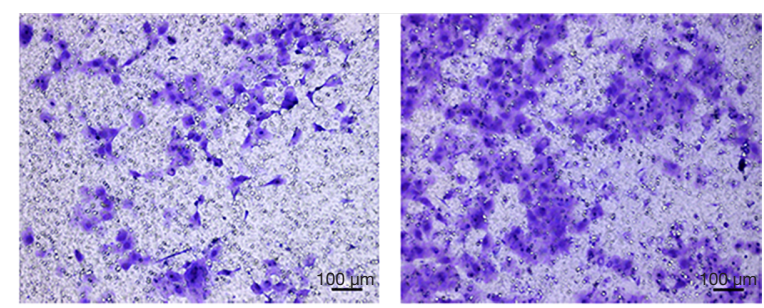

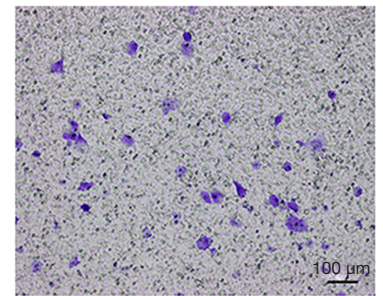

Monoculture

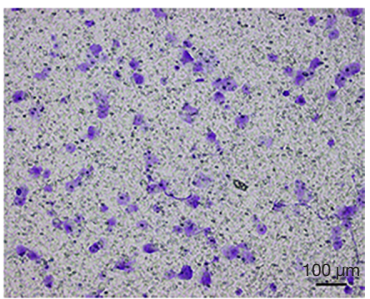

Coculture
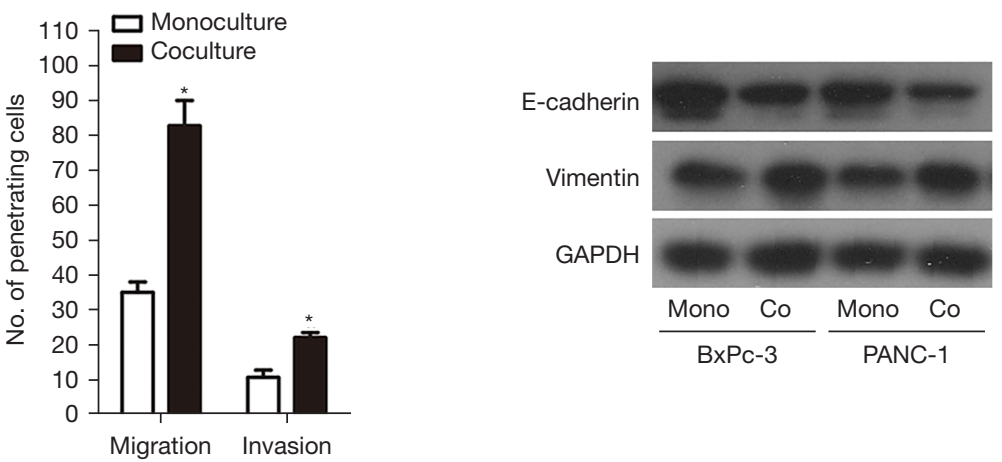

D

Figure 3 THP-1 monocytes promoted the invasiveness and EMT switch of PC cells. (A) Phase contrast microscopy showed a change in morphology for PANC-1 and BxPC-3 cell lines. Both PANC-1 and BxPC-3 cells lost their cell-to-cell contact, became elongated, and branched together when cocultured with THP-1 monocytes. (B) Migratory and invasive abilities of PANC-1 and BxPC-3 cells coculturing with THP-1 monocytes were evaluated by Transwell assay. The penetrating cells were stained with $0.05 \%$ crystal violet. (C) Histogram analysis for penetrating cells. (D) EMT-related markers (E-cadherin and vimentin) were detected by western blotting. THP-1 monocytes induced PANC-1 and BxPC-3 cells upregulation of vimentin while downregulating E-cadherin. Magnification: $100 \times$. Scale bars: 100 $\mu \mathrm{m}$. *, $\mathrm{P}<0.05$. GAPDH, glyceraldehyde 3-phosphate dehydrogenase; EMT, epithelial-mesenchymal transition; PC, pancreatic cancer. 
A

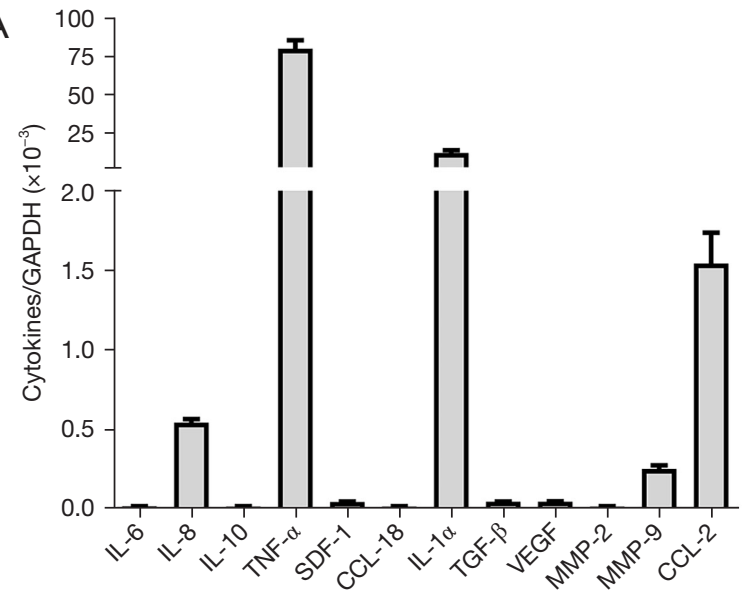

B

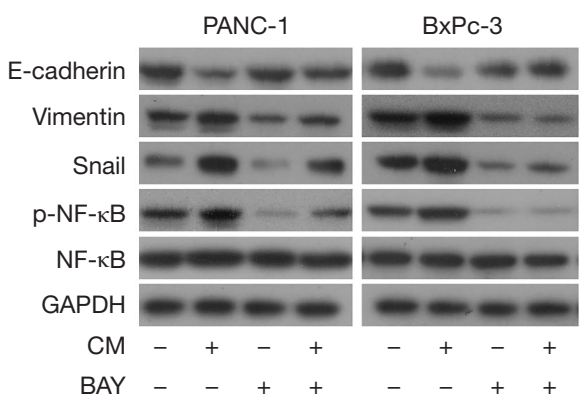

D
C
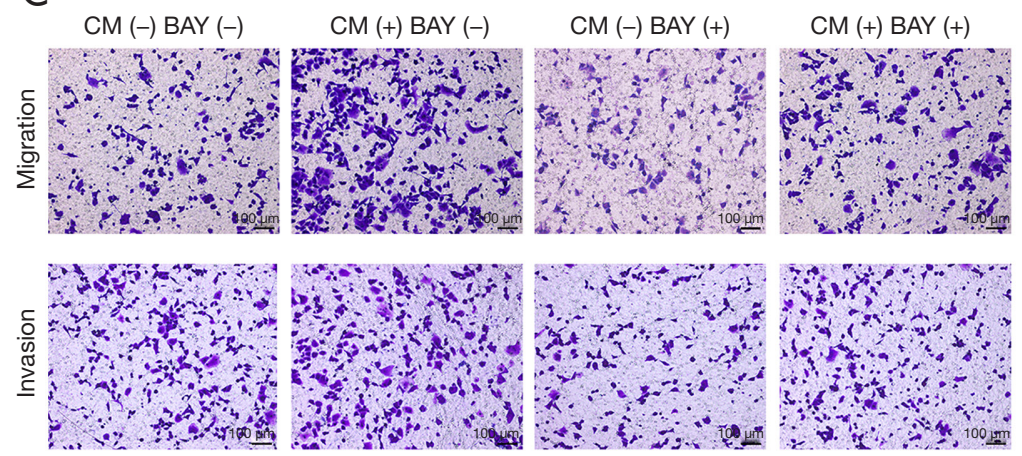

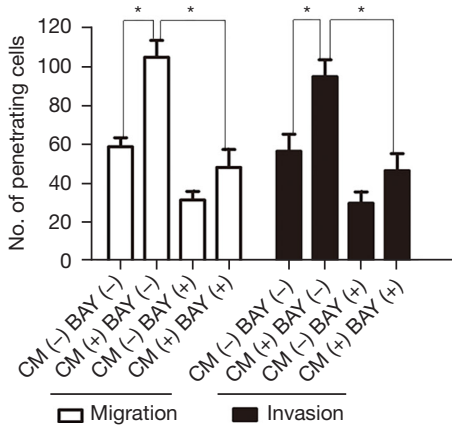

Figure 4 THP-1 monocytes promoted PC cells to undergo EMT switch via the NF- $\mathrm{BB}$ dependent upregulation of Snail. (A) qRT-PCR was performed to estimate a series of cytokines in THP-1 monocytes. TNF- $\alpha$ has demonstrated the highest expression of cytokine among

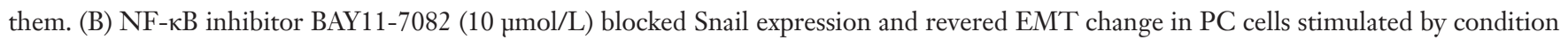
medium form THP-1 monocytes. CM: conditioned medium produced by THP-1 monocytes; BAY: BAY11-7082. (C,D) NF- $\kappa \mathrm{B}$ inhibitor BAY11-7082 (10 $\mu \mathrm{mol} / \mathrm{L})$ impaired PC cells migration and invasion abilities induced by conditioned medium from THP-1 monocytes. The penetrating cells were stained with $0.05 \%$ crystal violet. Magnification: $100 x$. Scale bars: $100 \mu \mathrm{m} .{ }^{*}, \mathrm{P}<0.05$. GAPDH, glyceraldehyde 3 -phosphate dehydrogenase; TNF- $\alpha$, tumor necrosis factor- $\alpha$; IL- $1 \alpha$, interleukin- $\alpha$; TGF- $\beta$, transforming growth factor- $\beta$; NF- $\kappa \mathrm{B}$, nuclear factor- $\kappa \mathrm{B}$; PC, pancreatic cancer; EMT, epithelial-mesenchymal transition; qRT-PCR, quantitative real-time polymerase chain reaction.

correlation analysis based on The Cancer Genome Atlas (TCGA) and the Genotype-Tissue Expression (GTEx) projects. As shown in Figure 5C,5D, the RNA sequencing expression level of Snail had positive correlation with cluster of differentiation 14 (CD14, marker of monocyte) (Spearman rank correlation coefficients, $r=0.540, \mathrm{P}<0.001$, Figure 5C), and CD14 also had positive correlation with CD68 (marker of macrophage) (Spearman rank correlation coefficients, $\mathrm{r}=0.650, \mathrm{P}<0.001$, Figure $5 D$ ). Further, a high expression of Snail was correlated with poor prognosis of $\mathrm{PC}$ as indicated by the survival analysis (high expression group: median survival time $=9$ months; low expression group: median survival time $=13$ months; log-rank test $\mathrm{P}=0.024$; Figure $5 E$ ). Similarly, both a higher expression of Snail and monocytes was associated with shorter overall survival compared with lower expressed participants ( 8 vs. 15 months, log-rank test $\mathrm{P}=0.002$; Figure $5 F$ ). These clinical findings illustrated that a high expression of Snail might be related to high absolute monocyte count and poor prognosis in PC.

\section{Discussion}

The tumor inflammatory microenvironment plays a great role in cancer initiation and progression. The systemic 
A
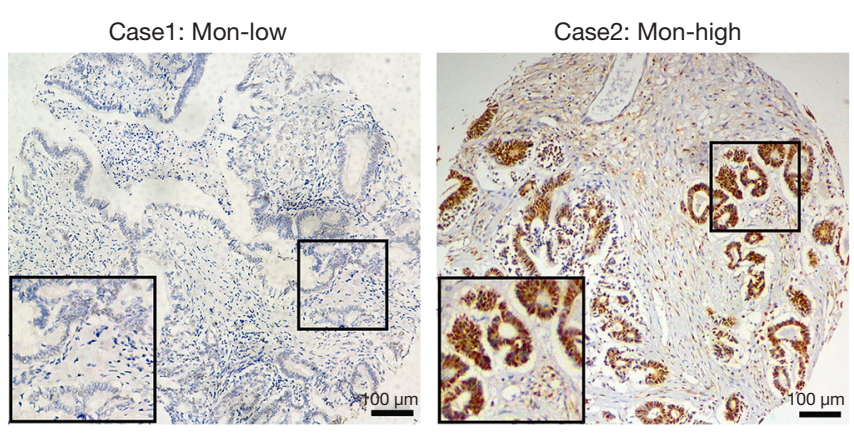

B

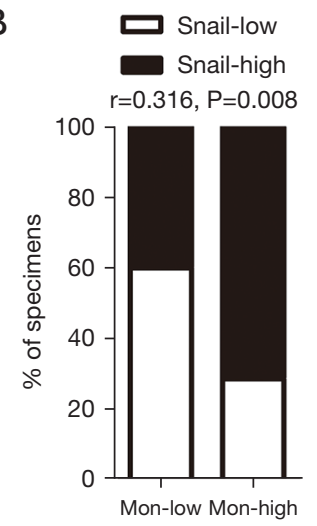

C

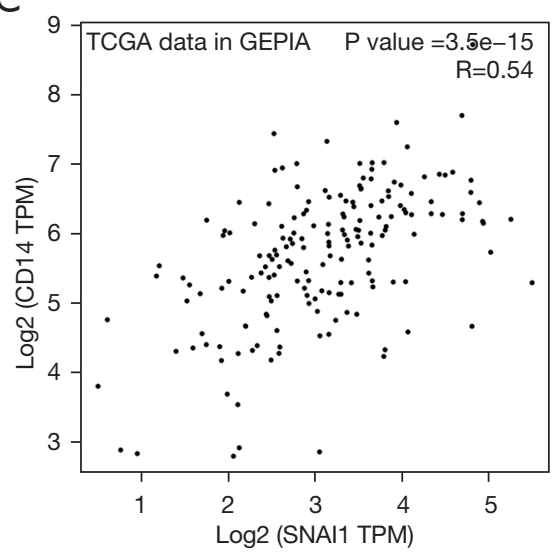

E

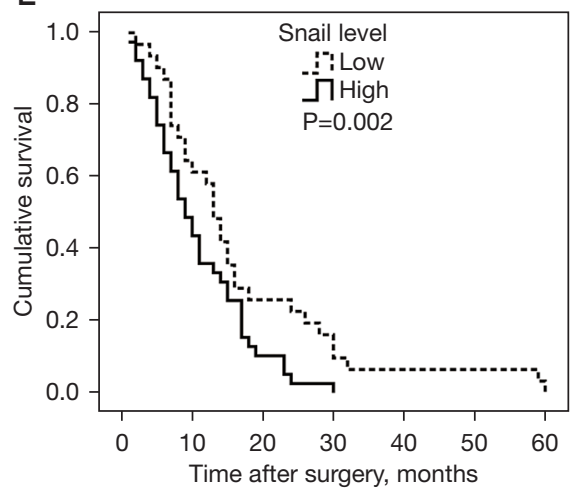

D

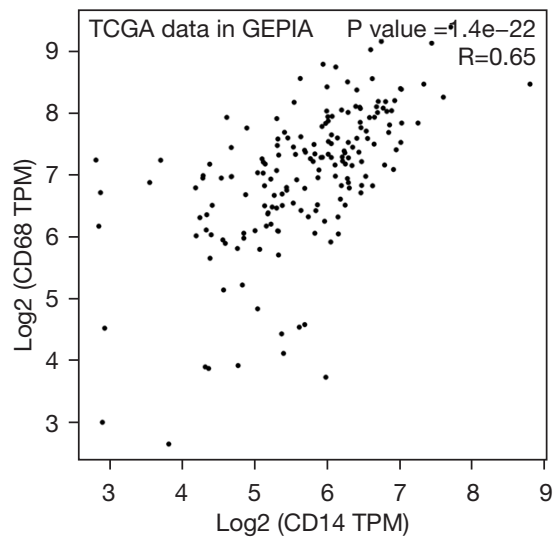

$\mathrm{F}$

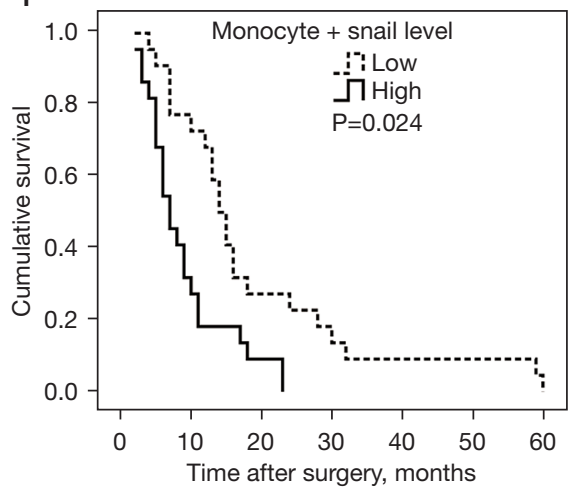

Figure 5 The expression level of Snail was correlated with peripheral blood monocyte count and the patients' outcome. (A) Representative images of IHC staining for Snail in PC tissue with high and low monocyte count. Magnification: 100x. Scale bars: $100 \mu \mathrm{m}$. (B) Percentage of samples showed low and high Snail expression relative to peripheral blood monocyte count. (C) The scatter plot demonstrated the correlation between the expression of Snail and CD14 by the GEPIA online tool. (D) Scatter plot demonstrated the correlation between the expression of CD14 and CD68 by the GEPIA online tool. (E,F) Correlation between overall survival of PC patients and Snail (E) expression, or both Snail and monocyte count (F). The Kaplan-Meier method and log-rank test were used for analysis. Expression level of: Snail-low ( $\mathrm{n}=31)$, Snail-high $(\mathrm{n}=39)$; Snail-low + low-monocyte count $(\mathrm{n}=21)$, Snail-high + high-monocyte count $(\mathrm{n}=25)$. TCGA, The Cancer Genome Atlas; IHC, immunohistochemical; PC, pancreatic cancer; CD14, cluster of differentiation 14; GEPIA, Gene Expression Profiling Interactive Analysis. 
response against malignant tumors coordinated by immune cells is associated with rearrangements of multiple hematological components, such as white blood cells, particularly the neutrophils, lymphocytes and monocytes. Immune cells and cancer cells build a reciprocal and mutually beneficial relationship to alleviate cancer growth and metastasis. Recently, increasing evidence highlighted that variables derived from the peripheral blood count (e.g., LMR) could be seen as a simple index of the immune function and may have a prognostic value in patients with solid tumors $(16,17)$.

In PC, many investigations have demonstrated that low LMR and high monocyte counts are predictors of poor patient long-term survival $(6,18)$; however, their detailed mechanisms of action are not fully understood. Our study found that elevated peripheral blood monocyte count was associated with poor survival and lymph node metastasis in PC, which aligned with other researches $(6,7,19)$. Some researchers have speculated several potential biological mechanisms linking monocyte with cancer biology (20). First of all, myeloid-derived suppressor cells (MDSCs) are recognized as a subpopulation of myeloid cells associated with tumor growth and progression (21). Monocyte-derived MDSCs (M-MDSCs) display an immune cell subset with profound immunosuppression function, and it is confirmed that targeting various components of M-MDSCs provides a promising future for the treatment of $\mathrm{PC}(9,22,23)$. Secondly, circulating blood monocytes migrate into cancer tissue and differentiate into TAMs after stimulating all kinds of cytokines (8). Then, TAMs regulate PC progression by promoting angiogenesis, metastasis, and pro-tumor immune response (10). Recently, Juusola et al. reported that peripheral blood monocytes displayed aberrant activation and immune suppression and had a tendency to differentiate into macrophages with pro-tumor function when stimulated by PC cells (24). Thirdly, the CCL2/CCR2 chemokine axis plays an important role in recruiting peripheral blood monocyte to the tumor microenvironment, and blockade of CCR2 restores anti-tumor immunity (25). It is reported that CCR2 inhibition is able to prevent liver metastasis and the CCR2 inhibitor PF-04136309 in combination with FOLFIRINOX chemotherapy is safe and tolerable for PC patients $(7,26)$.

In our study, we used a coculture system to explore the effect of monocytes on PC cells. Our results revealed that THP-1 monocytes could enhance PC cell migration and invasion through EMT switch, and we further revealed that the mechanism was THP-1 monocytes secreted some cytokines (such as TNF- $\alpha$ and IL-1 $\alpha$ ) to upregulate Snail expression via activating the NF- $\kappa \mathrm{B}$ signaling pathway. Baran et al. also reported that human peripheral blood monocytes secreted TNF- $\alpha$ to facilitate PC cell (HPC-4) motility via an EMT-dependent mechanism (27), which suggested that TNF- $\alpha$ was a vital cytokine secreted by monocytes. As a strong activator of the NF- $\mathrm{KB}$ signaling pathway, TNF- $\alpha$ is known to be constitutively activated in most PC patients (28) and has been shown to play key roles in the development of cancer (29). Reversely, many pro-inflammatory cytokines (like TNF- $\alpha$ ) target the genes of NF- $\mathrm{KB}$, and in doing so, form a positive feedback loop in the tumor inflammatory microenvironment. Liou et al. recently discovered that macrophages secreted RANTES (regulated on activation, normal $\mathrm{T}$ cell expressed and secreted) and TNF- $\alpha$ to activate NF- $\mathrm{NB}$ and its target genes, such as MMP-9 in the evolution of acinar to ductal metaplasia and pancreatic intraepithelial neoplasia (30). In addition, NF- $\kappa \mathrm{B}$ is involved in transforming growth factor- $\beta$ (TGF- $\beta$ )- or insulin-like growth factor binding protein 2 (IGFBP2)-induced EMT-phenotype in PC with upregulation of vimentin and ZEB1, and downregulation of E-cadherin (31,32). Meanwhile, NF- $\kappa \mathrm{B}$-activated monocytes produced Sonic hedgehog (Shh), contributing to PC progression (33). Collectively, these data strongly indicated a contribution of the NF- $\mathrm{\kappa B}$ signaling pathway in the interaction between cancer cells and stromal cells.

We also found that THP-1 monocytes accelerated PC cells to undergo EMT switch via the NF- $\kappa \mathrm{B}$ dependent up-regulation of Snail. The NF-кB/Snail-induced EMT phenotype was confirmed in other malignant tumors, such as colorectal cancer (34), glioma (35), lung cancer (36), lymphoma (37) and melanoma (38). Snail is a member of the Snail superfamily with domains containing zinc finger proteins and a key transcription factor of EMT (39). Positive expression of Snail was found in most PC tissues and associated with invasive characteristics $(40,41)$. In our study cohort, high expression of Snail was correlated with poor prognosis and elevated peripheral blood monocytes count.

Moreover, Snail acetylation promoted cancer cells to secrete CCL-2 and CCL-5 to recruit TAMs and induce macrophages to exhibit an M2-like phenotype (42). At the same time, TAMs could activate Snail expression by secreting different cytokines $(43,44)$. Therefore, a wide variety of evidence has pointed to a critical role of the $\mathrm{TNF}-\alpha / \mathrm{NF}-\kappa \mathrm{B} /$ Snail pathway in linking inflammation and cancer. 
This study had some potential limitations. First, the study cohort size was small, and there was selection bias, leading to restricted power to detect statistical differences on account of the retrospective nature of this study. Second, we used monocytoid THP-1 cells instead of human peripheral blood monocytes in the in vitro assays and did not analyze the difference of monocyte subgroups. A recent report demonstrated that the frequency of CD14+ CD16- HLADR ${ }^{\text {hi }}$ monocytes is a strong predictor of progression-free and overall survival in response to antiPD-1 immunotherapy in stage IV melanoma (45), which suggested that different subgroups of monocytes may have different biological functions.

In conclusion, our study highlighted that an increase in peripheral blood monocyte was a poor prognostic marker and was related to the progression of $\mathrm{PC}$ by promoting activation of the NF- $\mathrm{BB}$ signaling pathway, which in turn induced the Snail-dependent EMT switch to initiate cancer metastasis. Also, as the monocyte count is readily available in clinical practice through a simple routine blood test, it could be used as an easy and rapid indicator to assess tumor metastasis and patient survival.

\section{Acknowledgments}

Funding: This work was supported by the National Natural Science Foundation of China (Grant No. 81874057, 81972741, 82103142), Natural Science Foundation of Guangdong Province, China (Grant No. 2018A030310227), Fundamental Research Funds for the Central University (Grant No. 18zxxt59), Science and Technology Program of Guangzhou, China (Grant No. 202102020082), Medical Scientific Research Foundation of Guangdong Province, China (Grant No. A2018012, A2019447), Medical Science Foundation of Sun Yat-sen University (Grant No. 19ykpy109) and Guangdong Science and Technology Department (Grant No. 2017B030314026).

\section{Footnote}

Reporting Checklist: The authors have completed the MDAR reporting checklist. Available at https://dx.doi. org/10.21037/tcr-21-980

Data Sharing Statement: Available at https://dx.doi. org/10.21037/tcr-21-980

Conflicts of Interest: All authors have completed the ICMJE uniform disclosure form (available at https://dx.doi. org/10.21037/tcr-21-980) and report this work was supported by the National Natural Science Foundation of China (Grant No. 81874057, 81972741, 82103142) and Science and Technology Program of Guangzhou, China (Grant No. 202102020082). The authors have no other conflicts of interest to declare.

Ethical Statement: The authors are accountable for all aspects of the work in ensuring that questions related to the accuracy or integrity of any part of the work are appropriately investigated and resolved. The study was conducted in accordance with the Declaration of Helsinki (as revised in 2013). The study was approved by the Ethics Committee of Sun Yat-sen Memorial Hospital, Sun Yatsen University (SYSEC-KY-KS-2018-062) and individual consent for this retrospective analysis was waived.

Open Access Statement: This is an Open Access article distributed in accordance with the Creative Commons Attribution-NonCommercial-NoDerivs 4.0 International License (CC BY-NC-ND 4.0), which permits the noncommercial replication and distribution of the article with the strict proviso that no changes or edits are made and the original work is properly cited (including links to both the formal publication through the relevant DOI and the license). See: https://creativecommons.org/licenses/by-nc-nd/4.0/.

\section{References}

1. Siegel RL, Miller KD, Fuchs HE, et al. Cancer Statistics, 2021. CA Cancer J Clin 2021;71:7-33.

2. Chinese Pancreatic Surgery Association, Chinese Society of Surgery, Chinese Medical Association. Guidelines for the diagnosis and treatment of pancreatic cancer in China (2021). Zhonghua Wai Ke Za Zhi 2021;59:561-77.

3. Weisbeck A, Jansen RJ. Nutrients and the Pancreas: An Epigenetic Perspective. Nutrients 2017;9:283.

4. Kota J, Hancock J, Kwon J, et al. Pancreatic cancer: Stroma and its current and emerging targeted therapies. Cancer Lett 2017;391:38-49.

5. Tanaka HY, Kano MR. Stromal barriers to nanomedicine penetration in the pancreatic tumor microenvironment. Cancer Sci 2018;109:2085-92.

6. Sierzega $M$, Lenart $M$, Rutkowska $M$, et al. Preoperative Neutrophil-Lymphocyte and Lymphocyte-Monocyte Ratios Reflect Immune Cell Population Rearrangement in Resectable Pancreatic Cancer. Ann Surg Oncol 
2017;24:808-15.

7. Sanford DE, Belt BA, Panni RZ, et al. Inflammatory monocyte mobilization decreases patient survival in pancreatic cancer: a role for targeting the CCL2/CCR2 axis. Clin Cancer Res 2013;19:3404-15.

8. Wynn TA, Chawla A, Pollard JW. Macrophage biology in development, homeostasis and disease. Nature 2013;496:445-55.

9. Pergamo M, Miller G. Myeloid-derived suppressor cells and their role in pancreatic cancer. Cancer Gene Ther 2017;24:100-5.

10. Mielgo A, Schmid MC. Impact of tumour associated macrophages in pancreatic cancer. BMB Rep 2013;46:131-8.

11. Chen SJ, Zhang QB, Zeng LJ, et al. Distribution and clinical significance of tumour-associated macrophages in pancreatic ductal adenocarcinoma: a retrospective analysis in China. Curr Oncol 2015;22:e11-9.

12. Xu Y, Chang R, Peng Z, et al. Loss of polarity protein AF6 promotes pancreatic cancer metastasis by inducing Snail expression. Nat Commun 2015;6:7184.

13. Liu C, Cheng H, Jin K, et al. Application of the Eighth Edition of the American Joint Committee on Cancer Staging for Pancreatic Adenocarcinoma. Pancreas 2018;47:742-7.

14. Auwerx J. The human leukemia cell line, THP1: a multifacetted model for the study of monocytemacrophage differentiation. Experientia 1991;47:22-31.

15. Tang Z, Li C, Kang B, et al. GEPIA: a web server for cancer and normal gene expression profiling and interactive analyses. Nucleic Acids Res 2017;45:W98-W102.

16. Proctor MJ, Morrison DS, Talwar D, et al. A comparison of inflammation-based prognostic scores in patients with cancer. A Glasgow Inflammation Outcome Study. Eur J Cancer 2011;47:2633-41.

17. McMillan DC. Systemic inflammation, nutritional status and survival in patients with cancer. Curr Opin Clin Nutr Metab Care 2009;12:223-6.

18. Fujiwara Y, Misawa T, Shiba H, et al. Postoperative peripheral absolute blood lymphocyte-to-monocyte ratio predicts therapeutic outcome after pancreatic resection in patients with pancreatic adenocarcinoma. Anticancer Res 2014;34:5163-8.

19. Li GJ, Xu HW, Ji JJ, et al. Prognostic value of preoperative lymphocyte-to-monocyte ratio in pancreatic adenocarcinoma. Onco Targets Ther 2016;9:1085-92.

20. Tadmor T. Does monocyte count have prognostic significance in cancer? Leuk Res 2013;37:1193-4.
21. Talmadge JE, Gabrilovich DI. History of myeloid-derived suppressor cells. Nat Rev Cancer 2013;13:739-52.

22. Greten TF. Myeloid-derived suppressor cells in pancreatic cancer: more than a hidden barrier for antitumour immunity? Gut 2014;63:1690-1.

23. Panni RZ, Sanford DE, Belt BA, et al. Tumor-induced STAT3 activation in monocytic myeloid-derived suppressor cells enhances stemness and mesenchymal properties in human pancreatic cancer. Cancer Immunol Immunother 2014;63:513-28.

24. Juusola M, Kuuliala K, Kuuliala A, et al. Pancreatic cancer is associated with aberrant monocyte function and successive differentiation into macrophages with inferior anti-tumour characteristics. Pancreatology 2021;21:397-405.

25. O'Connor T, Borsig L, Heikenwalder M. CCL2-CCR2 Signaling in Disease Pathogenesis. Endocr Metab Immune Disord Drug Targets 2015;15:105-18.

26. Nywening TM, Wang-Gillam A, Sanford DE, et al. Targeting tumour-associated macrophages with CCR2 inhibition in combination with FOLFIRINOX in patients with borderline resectable and locally advanced pancreatic cancer: a single-centre, open-label, dose-finding, nonrandomised, phase 1b trial. Lancet Oncol 2016;17:651-62.

27. Baran B, Bechyne I, Siedlar M, et al. Blood monocytes stimulate migration of human pancreatic carcinoma cells in vitro: the role of tumour necrosis factor - alpha. Eur J Cell Biol 2009;88:743-52.

28. Wang W, Abbruzzese JL, Evans DB, et al. The nuclear factor-kappa B RelA transcription factor is constitutively activated in human pancreatic adenocarcinoma cells. Clin Cancer Res 1999;5:119-27.

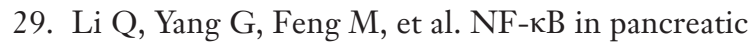
cancer: Its key role in chemoresistance. Cancer Lett 2018;421:127-34.

30. Liou GY, Döppler H, Necela B, et al. Macrophagesecreted cytokines drive pancreatic acinar-to-ductal metaplasia through NF- $\kappa \mathrm{B}$ and MMPs. J Cell Biol 2013;202:563-77.

31. Maier HJ, Schmidt-Strassburger U, Huber MA, et al. NF-kappaB promotes epithelial-mesenchymal transition, migration and invasion of pancreatic carcinoma cells. Cancer Lett 2010;295:214-28.

32. Gao S, Sun Y, Zhang X, et al. IGFBP2 Activates the NF- B Pathway to Drive Epithelial-Mesenchymal Transition and Invasive Character in Pancreatic Ductal Adenocarcinoma. Cancer Res 2016;76:6543-54.

33. Yamasaki A, Kameda C, Xu R, et al. Nuclear factor 
kappaB-activated monocytes contribute to pancreatic cancer progression through the production of Shh. Cancer Immunol Immunother 2010;59:675-86.

34. Feng M, Feng J, Chen W, et al. Lipocalin2 suppresses metastasis of colorectal cancer by attenuating NF- $\mathrm{kB}-$ dependent activation of snail and epithelial mesenchymal transition. Mol Cancer 2016;15:77.

35. Zhang B, Shi L, Lu S, et al. Autocrine IL-8 promotes $\mathrm{F}$-actin polymerization and mediate mesenchymal transition via ELMO1-NF- $\mathrm{kB}$-Snail signaling in glioma. Cancer Biol Ther 2015;16:898-911.

36. Chen KC, Chen CY, Lin CR, et al. Luteolin attenuates TGF- $\beta 1$-induced epithelial-mesenchymal transition of lung cancer cells by interfering in the PI3K/Akt-NF- $\mathrm{KB}-$ Snail pathway. Life Sci 2013;93:924-33.

37. Martinez-Paniagua MA, Vega MI, Huerta-Yepez S, et al. Galiximab signals B-NHL cells and inhibits the activities of NF- $\mathrm{kB}$-induced YY1- and snail-resistant factors: mechanism of sensitization to apoptosis by chemoimmunotherapeutic drugs. Mol Cancer Ther 2012;11:572-81.

38. Lin K, Baritaki S, Militello L, et al. The Role of B-RAF Mutations in Melanoma and the Induction of EMT via Dysregulation of the NF-kB/Snail/RKIP/PTEN Circuit. Genes Cancer 2010;1:409-20.

Cite this article as: Chen S, Huang F, He C, Li J, Chen S, Li Y, Chen Y, Lian G, Huang K. Peripheral blood monocytes predict clinical prognosis and support tumor invasiveness through NFB-dependent upregulation of Snail in pancreatic cancer. Transl Cancer Res 2021;10(11):4773-4785. doi: 10.21037/tcr-21-980
39. Moreno-Bueno G, Portillo F, Cano A. Transcriptional regulation of cell polarity in EMT and cancer. Oncogene 2008;27:6958-69.

40. Hotz B, Arndt M, Dullat S, et al. Epithelial to mesenchymal transition: expression of the regulators snail, slug, and twist in pancreatic cancer. Clin Cancer Res 2007;13:4769-76.

41. Chen J, Xu H, Zou X, et al. Snail recruits Ring1B to mediate transcriptional repression and cell migration in pancreatic cancer cells. Cancer Res 2014;74:4353-63.

42. Hsu DS, Wang HJ, Tai SK, et al. Acetylation of snail modulates the cytokinome of cancer cells to enhance the recruitment of macrophages. Cancer Cell 2014;26:534-48.

43. Fu XT, Dai Z, Song K, et al. Macrophage-secreted IL-8 induces epithelial-mesenchymal transition in hepatocellular carcinoma cells by activating the JAK2/ STAT3/Snail pathway. Int J Oncol 2015;46:587-96.

44. Kaler P, Galea V, Augenlicht L, et al. Tumor associated macrophages protect colon cancer cells from TRAILinduced apoptosis through IL-1beta-dependent stabilization of Snail in tumor cells. PLoS One 2010;5:e11700.

45. Krieg C, Nowicka M, Guglietta S, et al. High-dimensional single-cell analysis predicts response to anti-PD-1 immunotherapy. Nat Med 2018;24:144-53. 


\section{Supplementary}

\section{PANC-1}
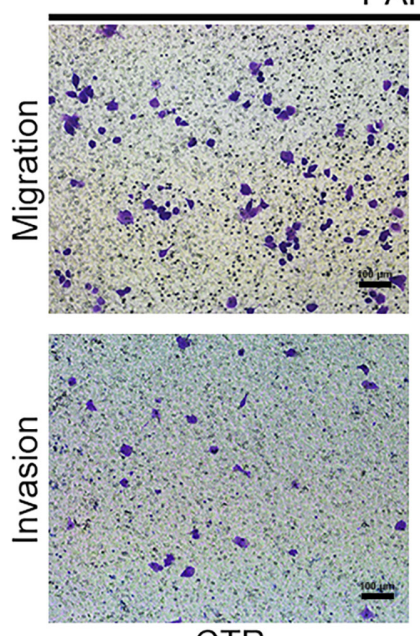

CTR
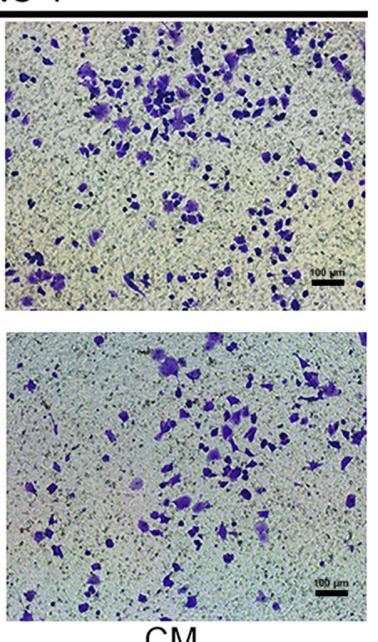
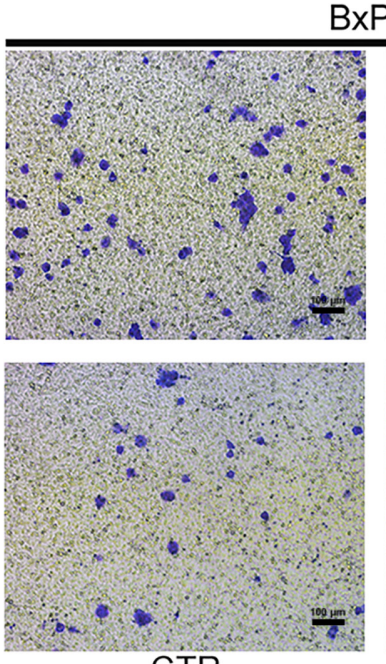

CTR

\section{BxPc-3}
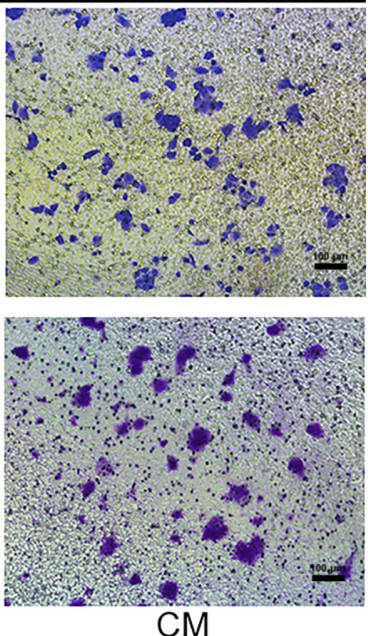

Figure S1 Conditioned medium from THP-1 monocytes promoted the invasion of PC cells. Magnification: 100x. Scale bars: $100 \mu \mathrm{m}$. CTR, control; CM, conditioned medium; PC, pancreatic cancer. 
Table S1 Primer sequences for qRT-PCR

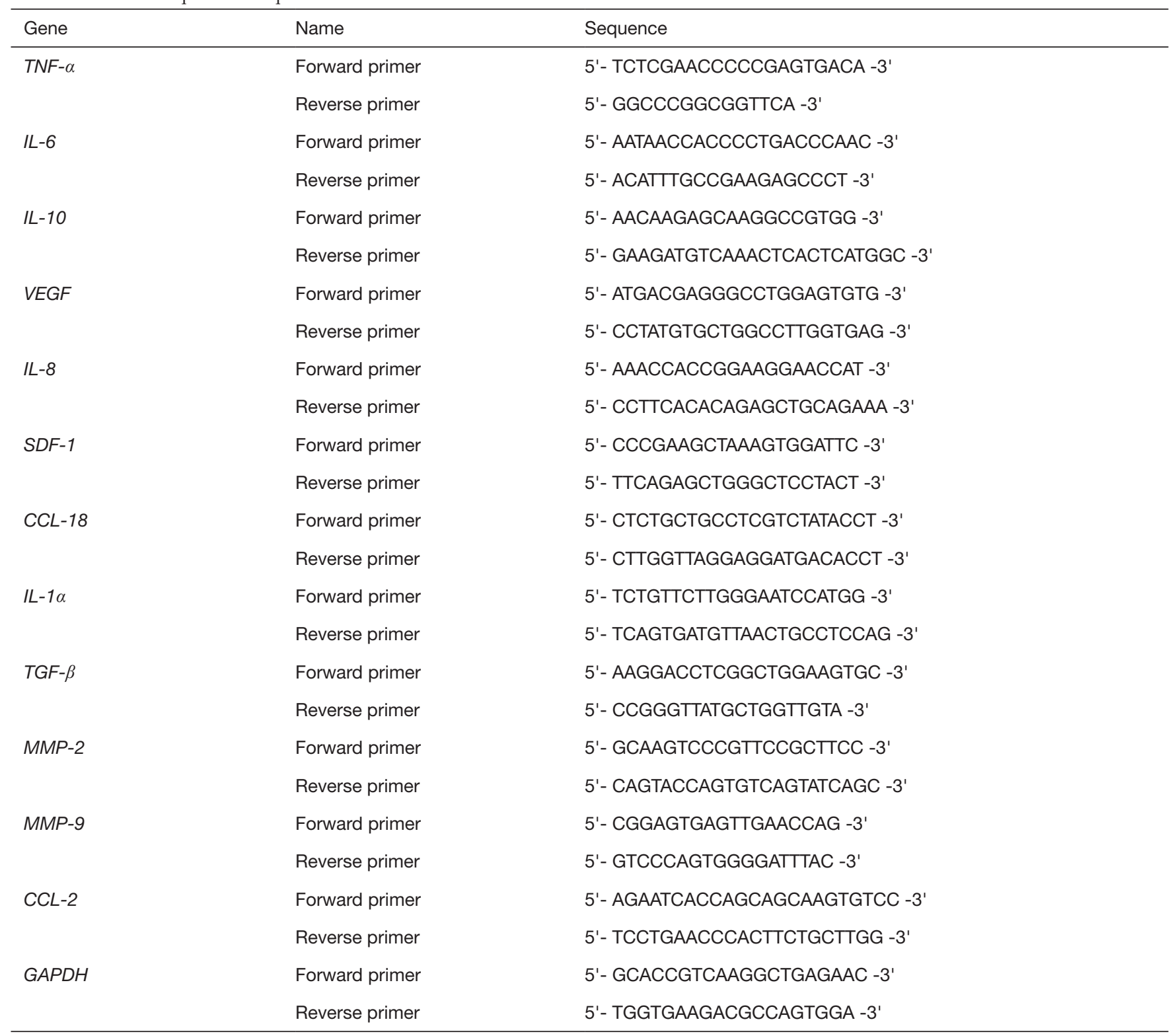

qRT-PCR, quantitative real-time polymerase chain reaction. 